\title{
System level co-simulation of a control valve and hydraulic cylinder circuit in a hydraulic percussion unit
}

\author{
Håkan Andersson ${ }^{1,2}$, Kjell Simonsson ${ }^{2}$, Daniel Hilding ${ }^{3}$, Mikael Schill ${ }^{3}$, and Daniel Leidermark ${ }^{2}$ \\ ${ }^{1}$ Construction Tools PC AB, Dragonvägen 2, 39127 Kalmar, Sweden \\ ${ }^{1}$ E-mail: hakan.andersson@se.atlascopco.com \\ ${ }^{2}$ Division of Solid Mechanics, Linköping University, 58183 Linköping, Sweden \\ ${ }^{3}$ DYNAmore Nordic AB, Brigadgatan 5, 58758 Linköping, Sweden
}

\begin{abstract}
In this study a previously developed co-simulation method that is based on a 1D system model representing the fluid components of a hydraulic machinery, within which structural 3D Finite Element (FE) models can be incorporated for detailed simulation of specific sub-models or complete structural assemblies, is further developed. The fluid system model consists of ordinary differential equation sub-models that are computationally very inexpensive, but still represents the fluid dynamics very well. The co-simulation method has been shown to work very well for a simple model representing a hydraulic driven machinery. A more complex model was set up in this work, in which two cylinders in the hydraulic circuit were evaluated. Such type of models, including both the main piston and control valves, are necessary as they represent the real application to a further extent than the simple model, of only one cylinder. Two models have been developed and evaluated, from the simple rigid body representation of the structural mechanics model, to the more complex model using linear elastic representation. The 3D FE-model facilitates evaluation of displacements, stresses, and strains on a local level of the model. The results can be utilised for fatigue assessment, wear analysis and for predictions of noise radiation.
\end{abstract}

Keywords: Co-simulation, Fluid-structure coupling, System simulation, Functional mockup interface, Fluid power machinery, Transmission line modelling

\section{Introduction}

The need of a computationally inexpensive co-simulation method for fluid power equipment and specifically oil hydraulic percussion units to achieve accurate predictions is of high importance to the industry. The end-product performance and fundamental mechanisms in these types of machines are highly affected by the fluid-structure interaction. The demands on such a simulation method are on one hand to facilitate the evaluation of global parameters but also to represent the detailed mechanisms at the fluid-structure interfaces. Global parameters must be evaluated from a system simulation model that often is built up by a network of sub-models represented by $1 \mathrm{D}$ components.

Detailed simulations of hydraulic machinery could be performed using existing Fluid-Structure Interaction (FSI) methods that today are available in many software suits, e.g. LS-DYNA [1] and ANSYS [2], where the fluid-, and the structural-mechanic systems are co-simulated. A few examples of these types of simulations from the machine building industry have been found, but are in general very rare. In the paper by Wang et al. [3] the valve system of a reciprocating air compressor was analysed through FSI-methods and in the paper by Campbell et al. [4] an FSI-analysis of a highly flexible impeller was conducted. A major drawback for the FSI-method is the need for extensive computational resources when solving these problems, especially when the models are used for system level simulations. Such computational resources are generally not available within the machine building industry, which requires less computationally demanding simulation methods to solve fluid-structure coupling problems.

It has been found that the functional behaviour of a product can be studied through system simulation models that are described by Ordinary Differential Equations (ODE), or 1D Partial Differential Equations (PDE) [5]. Simulation models based on Computational Fluid Dynamics (CFD) or the Finite Element Method (FEM) are used when phenomena's are studied at a much more detailed level. When combining these types of models proper boundary conditions can be provided at a low computational cost. In the work by Andersson et al. [6] such a co-simulation method is proposed that incorporates the fluid-structure coupling for a fluid power system. Here the fluid system is represented by a 1D system model, using the system simulation tool Hopsan [7], and the struc- 


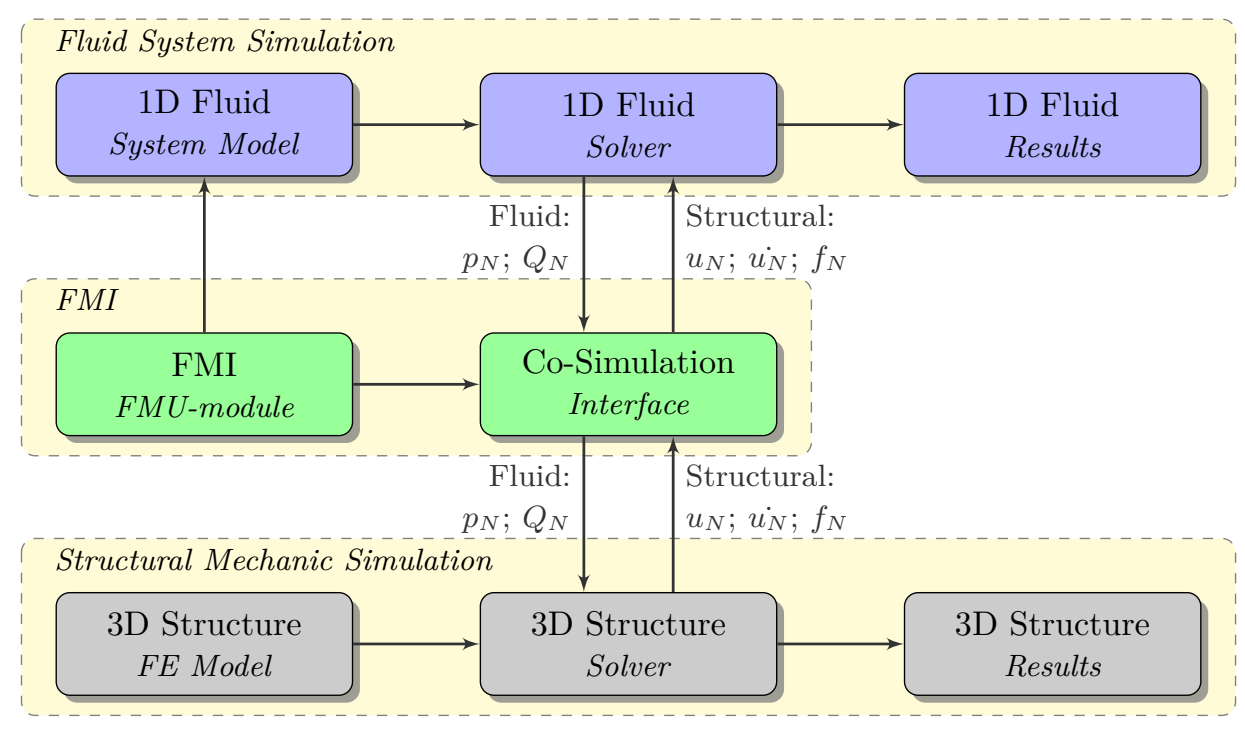

Figure 1: Overall simulation method sequence. The $N$ subscript indicates that multiple co-simulation sub-models are enabled

tural part is modelled in the Finite Element (FE) software LSDYNA. The models are then co-simulated using a communication module that is based on the Functional Mock-up Interface (FMI) standard [8], where a Functional Mock-up Unit (FMU) is defined on the system side to represent and connect to the FE-model. Using this technique the fluid system simulation will be computationally inexpensive but still reflect a correct dynamic behaviour, and it will also provide relevant input for the FE-simulation. This is particularly important for simulations over long time periods. Since the models are coupled it is also possible to evaluate the response from all fluid and structural loads.

In the literature, applications have been found where a 1D system model is co-simulated with a $3 \mathrm{D}$ model in the fluid or structural domain, to include global system behaviour into the 3D-model. These applications were often found to be of single domain simulations, i.e. fluid domain or structural domain, and consisting of two models communicating over a co-simulation interface. Two examples are found in [9], a 1D system model of ODE and a CFD-model in the fluid domain, and an example for the structural domain is found in [10], where a multi body simulation model and a 3D FE-model are co-simulated. These examples only consist of one sub-model for co-simulation and no example with multiple sub-models for co-simulation have been found. Furthermore, there are many other applications where a co-simulation setting is applicable, for instance, in fluid and multi-body mechanic interactions [11] and simulations based on meta-models [12].

Today's design tools for fluid and structural systems that are used for product development of hydraulic percussion units, are based on uncoupled analyses. This circumstance makes it very hard to capture coupled phenomenon at the design stage, and these are then first discovered during the prototype testing. At this stage it is time consuming and expensive to initiate redesign work that often can be quite extensive. In the previous paper by Andersson et al. [6] the proposed method was evaluated for one sub-model for co-simulation, repres-

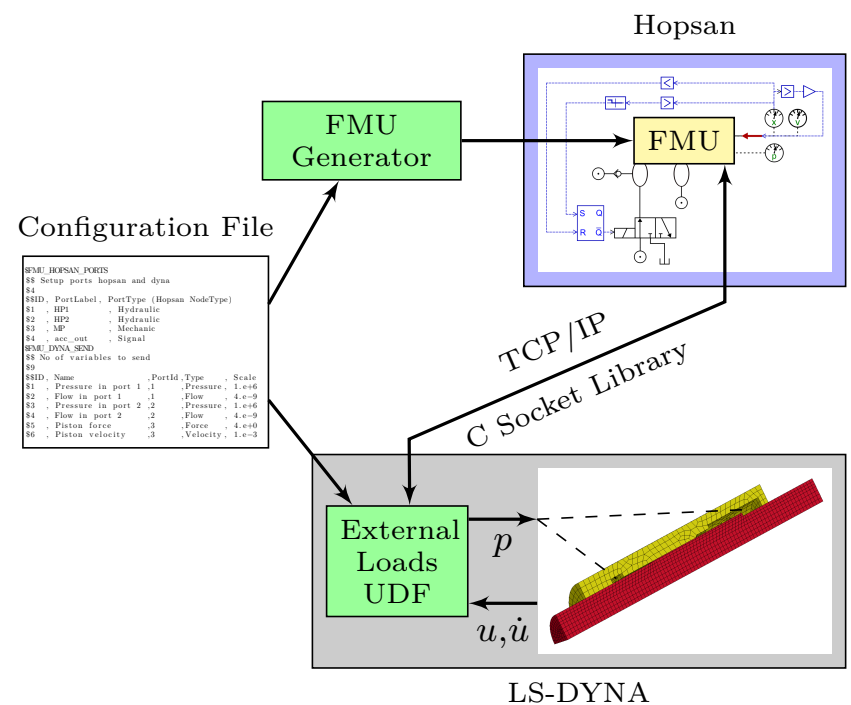

Figure 2: Overview of the co-simulation work flow

enting the structural part of a hydraulic cylinder. In order to achieve a more complete simulation model, see above, it was considered necessary to evaluate this method for multiple sub-models for co-simulation, as more accurate simulation models are continuously required. In the here presented work an FMU, which has been configured to contain multiple ports, is communicating with two hydraulic cylinders in the FE-model. The main hydraulic cylinder and the control valve, representing a hydraulically controlled piston with a hydraulic position feedback control signal. For these units the wave propagation and transmission in both fluid and structure are crucial and are often of short duration/high amplitude characteristic, and these data are transferred over the co-simulation interface that will be stressed to a high limit.

The outline of the paper is as follows. First, a brief presentation of the used simulation method and the previously im- 


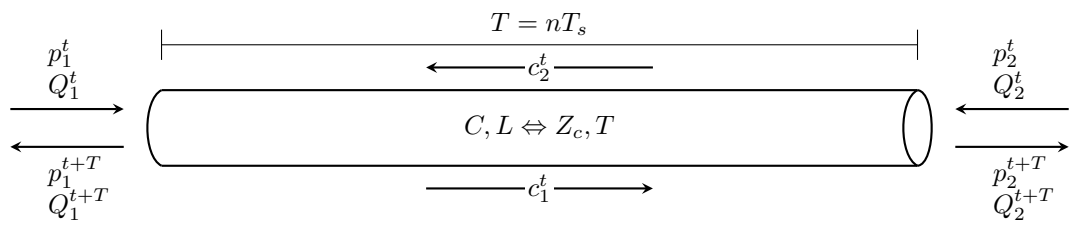

Figure 3: A TLM element, illustrated by a pipe, introduces a time delay $(T)$ of the effort $(p)$ and flow $(Q)$ variables entering on either side. Eq. 1, 2 and 3 gives the TLM boundary equations

plemented co-simulation interface is given. The application example and two different co-simulation cases are then explained and the accuracy of the method is evaluated and compared to an equivalent system-level simulation model.

\section{Simulation Method}

The simulation method that is used in this work is illustrated in Figure 1. As described by Andersson et al. [6] the implementation is based on the FMI-standard and a stand-alone software was developed to create and to configure the FMU that is used for the co-simulation interface. This software allows that several connection points for the FMU are defined. Thus, several ports for communication can be defined, which is here used to configure a co-simulation interface for two sub-models. The 1D system model consists of a number of sub-models describing different components in the hydraulic fluid system, e.g. valves, cavities, orifices etc.

In the FE-model, the fluid system is represented as actions on pressurised surfaces. From the fluid system model the pressure $p$ is delivered to the FE-model, while the displacement $u$, velocity $\dot{u}$ and mechanical force $f$ are received, see Figure 1. Since these values must be scalars in the 1D system, the average displacements and velocities over the pressurised surfaces are used.

At the end of the simulation the fluid domain variables are available and system properties such as performance and efficiency can be evaluated. From the FE-results it is possible to evaluate displacements and velocities of the 3D geometry, which can be used for instance as input data for acoustic analyses. The stresses and strains are also available e.g. for stress analysis and structural fatigue evaluation.

\section{The Co-simulation Implementation}

The same implementation as in the previous work by Andersson et al. [6] has also been used here, with the opensource 1D system-simulation program Hopsan [7, 13], for simulation of the hydraulic fluid components, and the commercial FE-software LS-DYNA [1], for the structural part. Hopsan is based on the Transmission Line Modelling (TLM) method for modelling of physical components, which has been shown to be suitable for simulation of hydro-mechanical systems [14]. In LS-DYNA the explicit solver is used as it is fast and appropriate for analysing stress waves in solid structures. The co-simulation interface is realised as an FMU on the Hopsan side and for LS-DYNA the User Defined Function (UDF) is used. Previous work by Larsson et al. [15], where they compared different integration methods for co-

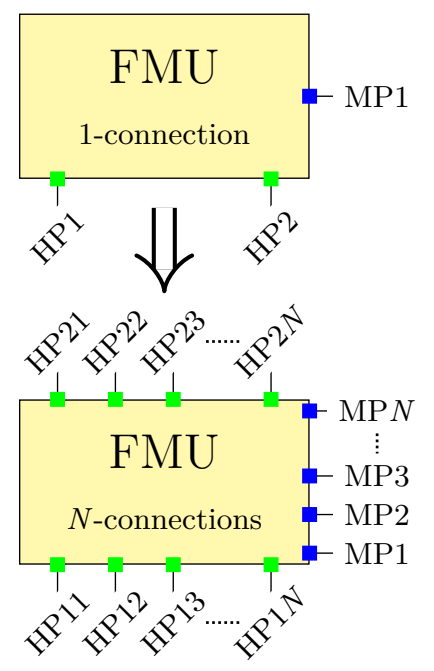

Figure 4: This figure shows the development of the simulation method to increase the number of sub-models for cosimulation. At the top is an FMU configured for one submodel. The FMU at the bottom is more general, containing $N$-number of sub-models. HPxx are the hydraulic ports and MPx are the mechanical ports

simulation, shows that co-simulation based on TLM offers very good numerical stability. The investigation showed that co-simulation based on TLM had superior stability properties. The numerical stability has not been further investigated in this work.

In order to achieve high performance and flexibility in communication the co-simulation interface uses the TCP/IP for transfer of data during the simulation.

A custom made configuration file based on the native Keyword format in LS-DYNA is utilised both to set-up the FMU and to configure the UDF in LS-DYNA. The FMU is built using an in-house developed automatic software generator. In the UDF the connection between the FE-model, nodes and element segments, and the hydraulic- or mechanial ports is specified. The co-simulation connectivity and work flow is illustrated in Figure 2.

A brief overview of the Hopsan simulation tool and the UDF is given below, for further details regarding this co-simulation implementation and other issues related to the used softwares, please see [6]. 


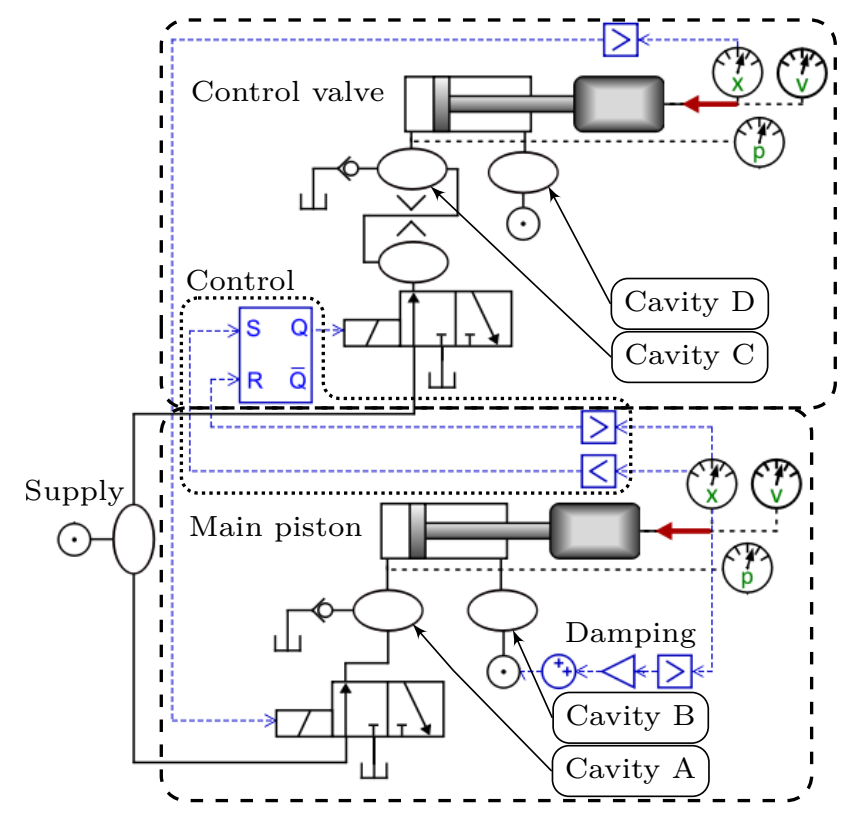

(a) Hopsan reference sytem model

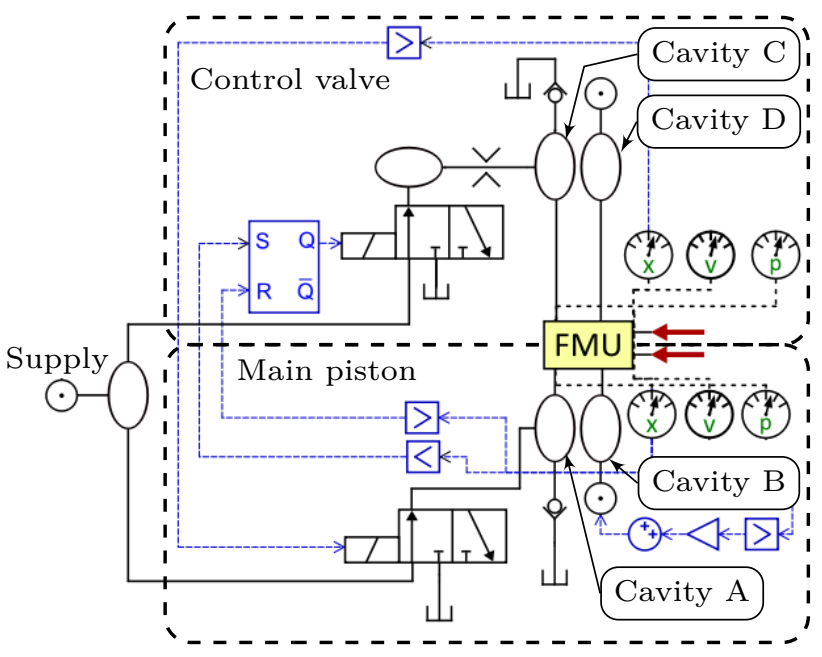

(b) Hopsan system model for co-simulation

Figure 5: System models for Hopsan simulations. The components for each hydraulic cylinder, the main piston and the control valve are encircled by dashed lines. The valve control is encircled by dotted line, i.e. Control, and the components for the piston damping mechanisms are indicated, i.e. Damping. The meters are sensors for different quantities; X=Displacement $u$, $V=$ Velocity $\dot{u}$ and $p=$ Pressure. $X$ and $V$ are Hopsan standard nomenclature, but the latter ones will be used throughout this work. The displacement and the velocity sensors are connected to the control valve and to the main piston. The pressure sensors are connected to Cavity $A$ in the main piston cylinder and to Cavity $C$ in the control valve cylinder

\subsection{The Hopsan Simulation Tool}

Hopsan is a free multi-domain 1D system simulation tool that uses the TLM method for modelling and simulation of physical systems. The TLM method, or bi-lateral delay line modelling, firstly presented by Auslander [16] in 1968, is an approach to model dynamic systems by a network of distributed sub-models, i.e. TLM elements. The core in this technique is that a time delay is introduced by using the one-dimensional wave equation in the element, which decouples the connection points and make it possible to solve each part separately. Since the connection points by the time delay are weakly coupled, explicit methods rather than iterative methods, can be used for solving the simulation model very effectively.

This co-simulation implementation is based on loss-less, single-time-step TLM elements, see Figure 3. The governing equations for the time-domain are given in Eq. 1, 2 and 3 , where $p_{1}$ and $p_{2}$ are the 'effort' variables and $Q_{1}$ and $Q_{2}$ are the 'flow' variables at the boundaries [17]. During the delay, waves represented by $c_{1}$ and $c_{2}$ travel in the respective directions through the element. The loss-less element's characteristic impedance $Z_{c}$ is a scalar property that relates the physical properties, capacitance $C$ and inductance $L$, to the introduced time delay $T$.

$$
\begin{aligned}
& p_{2}^{t+T}=\overbrace{p_{1}^{t}+Z_{c} Q_{1}^{t}}^{c_{1}^{t}}+Z_{c} Q_{2}^{t+T} \\
& p_{1}^{t+T}=\underbrace{p_{2}^{t}+Z_{c} Q_{2}^{t}}_{c_{2}^{t}}+Z_{c} Q_{1}^{t+T}
\end{aligned}
$$

$$
\begin{aligned}
& Z_{c}=\sqrt{\frac{L}{C}} \\
& T=\sqrt{L C}
\end{aligned}
$$

The pressure acting on the piston in the FE-model is calculated according to Eq. 4. As can be noticed from this equation the flow variable, $Q_{2}^{t}$, is taken from the previous time step, which is an approximation and a possible source of error. However, the difference for small time steps should not be of importance.

$$
p_{2, \text { approx }}^{t+T}=c_{1}^{t}+Z_{c} Q_{2}^{t}
$$

\subsection{LS-DYNA User Defined Function}

On the structural side, the general non-linear ODE, i.e. the equation of motion, that is solved by LS-DYNA is given by Eq. 5.

$$
\mathbf{M u ̈}+\mathbf{C u}+\mathbf{f}_{I n t}=\mathbf{f}_{E x t}
$$

where $\mathbf{M}$ is the mass matrix, $\mathbf{C}$ is the damping matrix and $\mathbf{f}_{I n t}$ is the internal force vector. The external force vector, $\mathbf{f}_{E x t}$, 


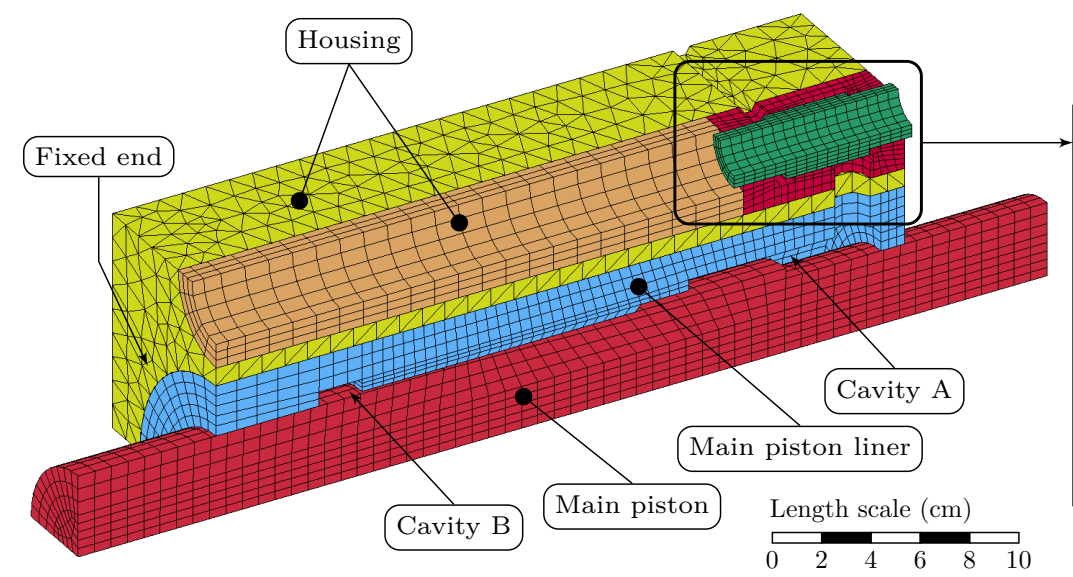

(a) Complete FE-model

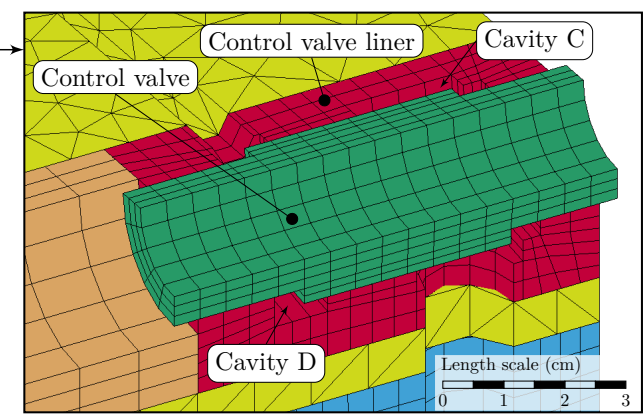

(b) Control valve details

Figure 6: The quarter FE-model that is used in Case 1.

represents all external loads acting on the FE-model. The input variables $\left(c\right.$ and $Z_{c}$ ), representing the pressure, from the system model are used to calculate the external loads $\mathbf{f}_{E x t}$ and the user defined configuration file specifies on which locations they are to be applied through the UDF. The displacement $\mathbf{u}$ is solved explicitly by the central difference time integration scheme described by Eq. 6 and 7. For further description regarding the explicit integration scheme see e.g. [18].

$$
\begin{gathered}
\dot{\mathbf{u}}^{n+\frac{1}{2}}=\dot{\mathbf{u}}^{n-\frac{1}{2}}+\ddot{\mathbf{u}}^{n} \Delta t^{n} \\
\mathbf{u}^{n+1}=\mathbf{u}^{n}+\dot{\mathbf{u}}^{n+\frac{1}{2}} \Delta t^{n+\frac{1}{2}}
\end{gathered}
$$

The resulting displacements and velocities are sent back from the FE-model to the system model, and this procedure is repeated for each time step until the analysis is completed.

\subsection{Multiple Components for Co-simulation}

As mentioned above, the main idea in this work was to implement co-simulation couplings for multiple sub-models, and has here been implemented and evaluated for two sub-models representing two hydraulic cylinders, and the FMU was configured to contain the necessary ports for these sub-models. This feature facilitates co-simulation of more than one component, or region, and it can be used for several important areas of fluid-structure interaction mechanisms in the simulation model.

Figure 4 shows a general picture where the FMU from the presented work of Andersson et al. [6] is shown at the top, which is configured for one sub-model. At the bottom is an FMU shown configured for an arbitrary number of interface ports. This implementation only supports one FMU on the Hopsan side and one FE-model, but the interface can handle more than one sub-model as long as it is connected to the same FMU and to the same FE-model.

The interface was evaluated for two sub-models and the upper limit of the number of connections is not examined. The FMU was reconfigured using the FMU-generator and the new ports were added in the configuration file. The system simulation model in Hopsan was extended to also include the necessary components for the control valve, which were connected to the added hydraulic- and mechanical ports on the redefined FMU. On the LS-DYNA side, the FE-model was redesigned to also include the structural parts of the control valve, and the element segments for each cavity were specified in the UDF.

\section{Simulations and Results}

As discussed above, a simple fluid power model of a hydraulic percussion unit was set-up in order to validate the co-simulation method for an extended number of hydraulic cylinders. This model contains two hydraulic cylinders, the first represents the main piston and the second is representing the control valve, which concludes the main structural parts that affects the fluid-structure coupling in a hydraulic percussion unit. The model reflects important features, such as short duration dynamics in the fluid and the structural systems.

A reference model was set-up in Hopsan, where the fluid system consists of two hydraulic cylinders, the necessary fluid volumes, a valve and the pressure supply component, see Figure 5a. The valves, controls the oil flow to each cylinder, while Cavity A - D represent the fluid properties of the oil volumes inside each cylinder, also shown in Figure 6. The fluid properties used for the hydraulic oil can be found in Table 1. A point mass, representing a rigid body, is connected to the piston part of each hydraulic cylinder. Each

Table 1: The fluid and solid material properties used in the simulations

\begin{tabular}{lcccc}
\hline Quantity & Material & Symbol & Unit & Value \\
\hline Bulk modulus & Oil & $K$ & $\mathrm{GPa}$ & 1.6 \\
Density & Oil & $\rho_{\text {oil }}$ & $\mathrm{kg} / \mathrm{m}^{3}$ & 890 \\
Elastic stiffness & Steel & $E$ & $\mathrm{GPa}$ & 210 \\
Poissons ratio & Steel & $v$ & - & 0.3 \\
Density & Steel & $\rho_{\text {steel }}$ & $\mathrm{kg} / \mathrm{m}^{3}$ & 7850 \\
\hline
\end{tabular}




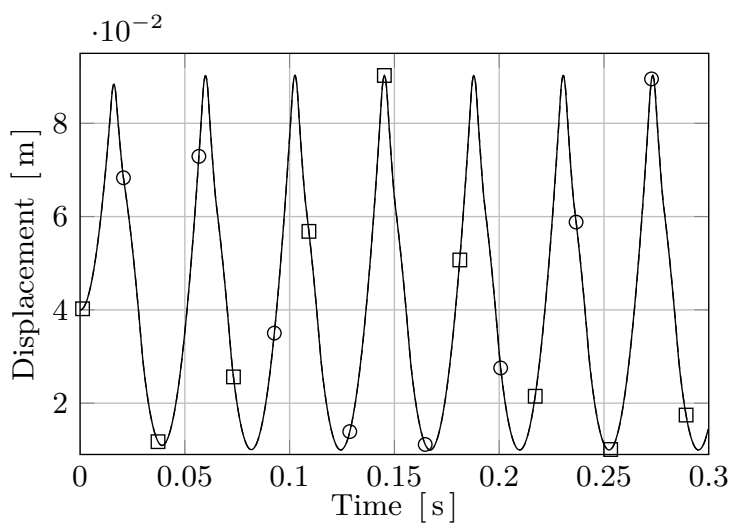

(a) Main piston displacement

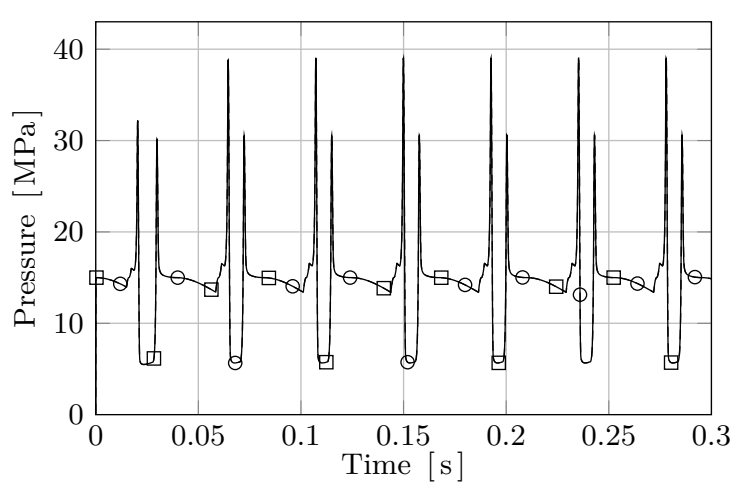

(c) Pressure in piston Cavity A

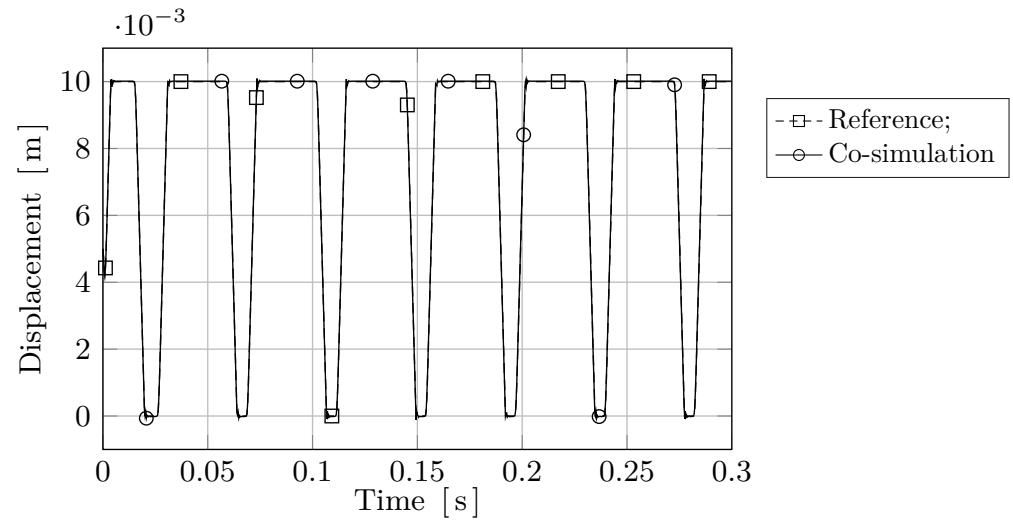

(b) Control valve displacement

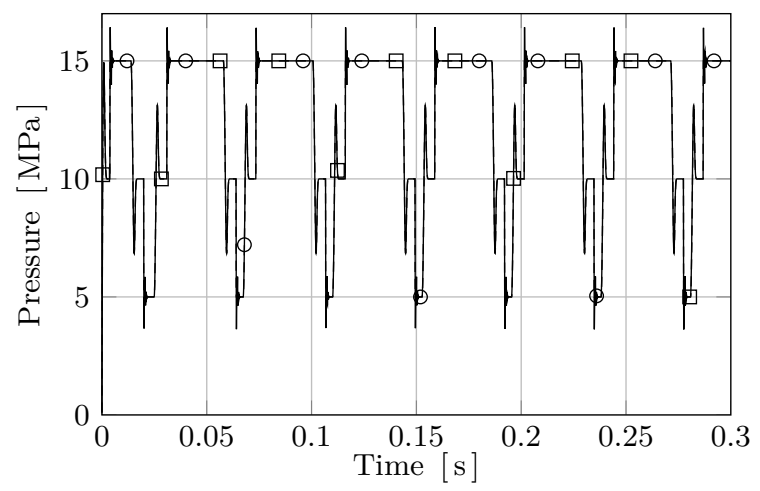

(d) Pressure in control valve Cavity C

\section{Figure 7: Simulation results from Case 1, showing the difference between the reference and the co-simulation models}

valve is controlled by a network of logical components, the control circuit, see Figure 5a. The position of the control valve controls the main piston valve and vice versa. Signals are generated at pre-determined positions for each cylinder to achieve the reciprocal movement for the main piston. A hydraulic damping mechanism for the main piston is used to prevent mechanical impact at the outer end position, see Damping in Figure 5a. This mechanism significantly increases the pressure above the normal level in Cavity B when the piston reaches a pre-set position that is located a small distance inside the mechanical end point. The increased pressure will cause the piston to stop and reverse inwards. When the piston is inside the pre-set position again the pressure will decrease to the normal level. Several sensors, see the meters in Figure 5, were used to collect data during the simulation. The displacement and the velocity sensors are connected to each of the pistons and the pressure sensors are connected to Cavity $\mathrm{A}$ and $\mathrm{C}$. The reference system model is completely simulated in Hopsan and its results are used as a reference during the evaluation of the co-simulation results. A second system model was defined for the co-simulation approach, see Figure $5 \mathrm{~b}$. This is identical to the reference model, except for the hydraulic cylinders. The cylinders have been replaced by the imported FMU. The FMU is for this example configured to contain interface ports for two cylinders. This system model uses the FE-model shown in Figure 6 for co-simulation with

\section{LS-DYNA.}

The FE-model of the hydraulic cylinder assembly consists of the main piston, the control valve, the liners and the housing parts. The mesh consists of 7211 8-node hexahedral- and 9855 4-node tetrahedral solid elements, which gives a total number of 17066 elements. All tetrahedral elements belong to the main housing. A full integration scheme was used for all solid elements. The liners and the housing parts are rigidly connected to each other by tied contacts in LS-DYNA. Typical elastic material properties for steel were used in the FE-model, see Table 1. Due to symmetry, a quarter model is used, and the associated boundary conditions are applied to the nodes on the symmetry planes. The main piston and the control valve are free to move in the axial direction, but due to symmetry constrained in all the other directions. The axial mechanical end points for these components are defined by surface to surface contact definitions. Oil is present at the contact surfaces that will dampen the contact forces. This behaviour is accounted for by introducing a viscous dampening for the contact definition. A factor of $60 \%$ of the critical viscous damping was applied. The axial movement of the cylinder housing is constrained due to the fixed boundary condition on the end surface and the other directions are constrained due to symmetry. The total mass for each part is defined by the FE-mesh and the material density. Since a quarter FE-model is used, a scaling routine implemented in the co-simulation in- 


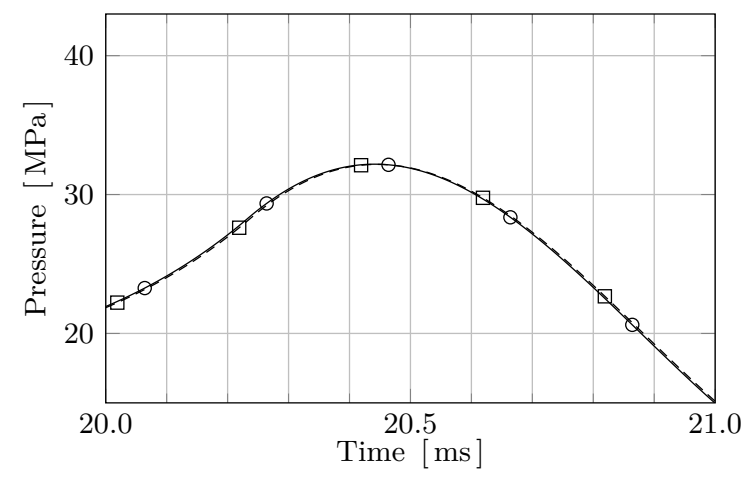

(a) First pressure peak

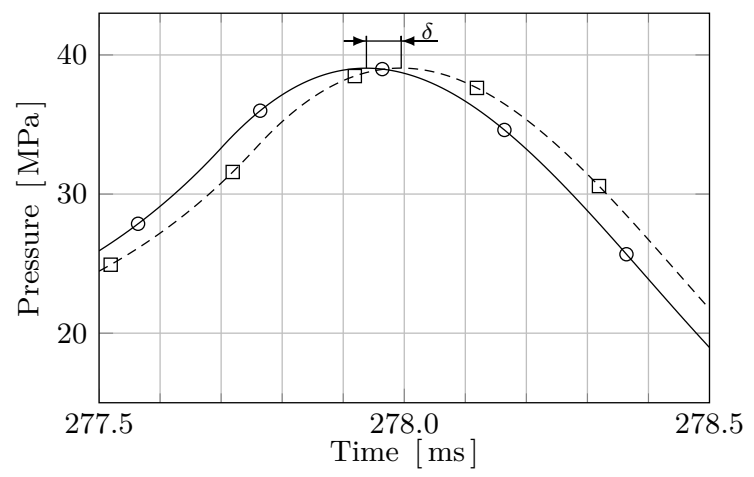

(b) Second last pressure peak

Figure 8: These curves are the same as in Figure 7c but shown at a much smaller time scale. A time period of 1.0 ms is displayed around $a)$ the first and $b$ ) the second last pressure peak. The parameter $\delta$ denotes the time shift between the two simulation systems

terface was utilised to get the equivalent full model response on the Hopsan side. Each cylinder has two hydraulic control surfaces that are associated with its respective cavity. The pressure, based on $c$ and $Z_{c}$, from the Hopsan simulation is applied to all element segments that belong to each cavity. A control routine keeps track of when the element segment on the moving part is inside or outside the cavity. When the segment is outside the cavity the pressure is removed, and when it has moved inside again the pressure is restored.

The start values for the simulation, at $t=0 \mathrm{~s}$, were as follows: main piston position $u=0.04 \mathrm{~m}$, control valve position $u=$ $0.005 \mathrm{~m}$, velocity and acceleration for the main piston and the control valve, $\dot{u}=\ddot{u}=0$, pressure in all cavities, $p=10^{5} \mathrm{~Pa}$. At the start of the simulation a pressure of $p=15 \mathrm{MPa}$ at the Supply sub-model will be transferred to each component in the hydraulic circuit. When the pressure increase reaches the main piston and the control valve, they will start to move and this event determines the starting point for the first working stroke, and the main piston will reach a steady state behaviour after a few working cycles.

The co-simulation method for two hydraulic cylinders has been evaluated through two different simulation cases. These are:

\section{Case 1 Hydraulically controlled main piston and control valve, with rigid body representations}

Case 2 Hydraulically controlled main piston and control valve with linear elastic material properties for the structural parts. Repeated impacts against an elastic cylinder is analysed

Case 1 represents, for the main piston, a moving mass that is purely driven by hydraulic pressure and no external loads are applied, and for the control valve the mass is moving between its mechanical end points. The contact mechanism is not modelled in Hopsan, but in LS-DYNA and will therefore give rise to a difference between the two simulation models. These forces will affect the motion of the valve at the end points. Case 2 gives a demonstration of the method when somewhat more realistic conditions, such as elastic materials and real contact definitions, are used. This makes it possible to simulate deformations, stresses and strains. For Case 1 the co-simulation results are investigated and compared with the results from the reference model in Hopsan. This is not possible for Case 2 since the impact mechanism between the piston and the tool can not be modelled using the standard components libraries in Hopsan.

The simulations were run on an Intel Xeon E5-1660 (3.0 GHz, 8 core) workstation with 32 GB RAM under Windows 7 (64bit). Eight processor cores with shared memory were used for the LS-DYNA analyses and one core for the Hopsan analyses. Both Hopsan and LS-DYNA were run on the same computer.

\subsection{Case 1}

This case simulates the effects when hydraulic pressure is applied to both the main piston and the control valve. The time step $T=10^{-6} \mathrm{~s}$ was used here based on a convergence study of the Hopsan reference model, cf. Fig. 5a. The method for this convergence study was as follows: The simulations were made using the following time step values, $T_{i}=10^{-i} \mathrm{~s}$. From each simulation, using the time step $T_{i}$, the piston displacement $u_{T_{i}}$, cf. Fig. 7a, was extracted and the Root Mean Square $(r m s)$ value for this signal was calculated, $u_{T_{i} r m s}$. The reference value, $u_{T_{i+1} r m s}$, was calculated from a simulation performed with a time step one decade shorter, i.e. $T_{i+1}$. The error $\varepsilon_{i}$ was defined according to Eq. 8. The procedure was repeated until the error level was below $0.1 \%$, which was considered acceptable for this application. An error level of $\approx 0.07 \%$ was reached for $i=6$, which gives a time step of $T=10^{-6}$ s.

$$
\varepsilon_{i}=\frac{u_{T_{i} r m s}}{u_{T_{i+1} r m s}}
$$

The parasitic masses for each hydraulic cavity were estimated and is found in Table 2. The parasitic masses were found to be very small and can be neglected in comparison to the mass of the main piston and the control valve. 


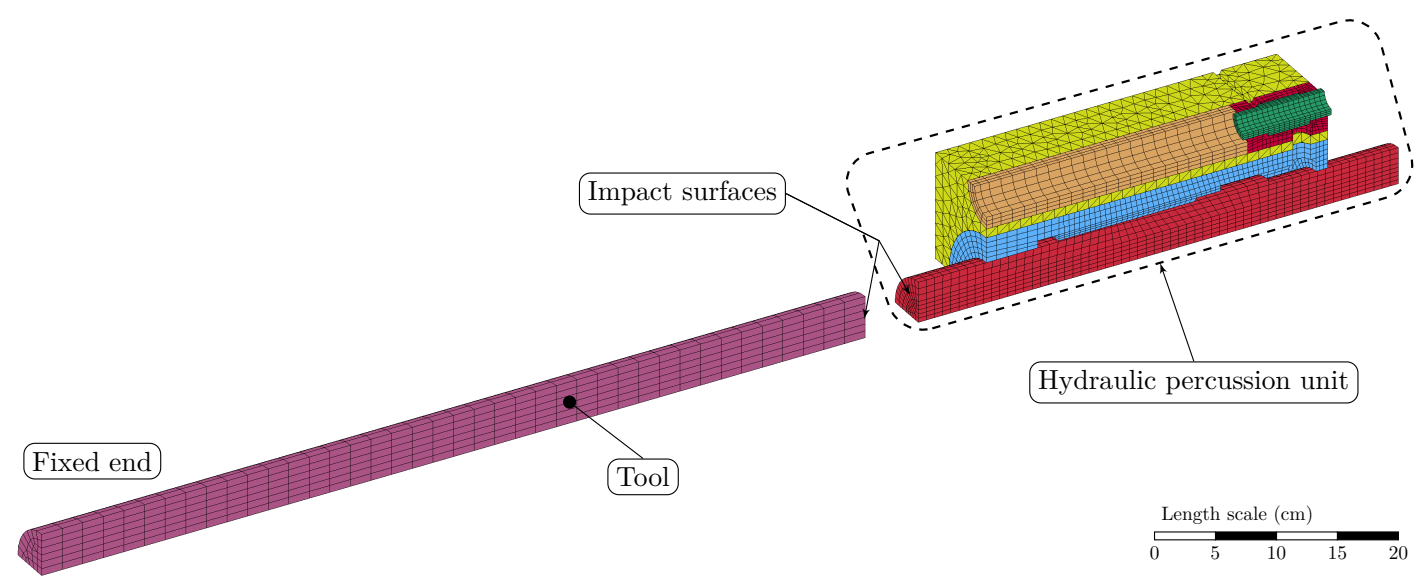

Figure 9: The FE-model used in Case 2. The hydraulic percussion unit is the same as in Case 1, see Figure 6, except for the elastic material properties. The left cylindrical part represents the tool. The left end of the tool is rigidly fixed and on the other surfaces symmetry conditions are applied

The computational time for the co-simulation was approximately 7 minutes and for the pure Hopsan reference model $0.3 \mathrm{~s}$. Simulation results are shown in Figure 7.

Figure $7 \mathrm{a}$ shows that the main piston moves in and out of the housing with a stroke length of $\sim 0.08 \mathrm{~m}$ at an impact frequency of $\sim 23 \mathrm{~Hz}$, and Figure $7 \mathrm{~b}$ shows that the control valve is moving $0.01 \mathrm{~m}$ between its mechanical end positions. It can be seen that the results for the reference and the co-simulation model coincide. This was to be expected since the two system models are the same except for the piston movement simulation. In Figure 7c it can be seen that the curves are right on top of each other, but when the curves are examined at a greater magnification, a maximum time shift of $\sim 57 \mu$ s can be noticed at the second last pressure peak, see Figure 8 . It was also found that the time shift is increasing throughout the simulation.

The pressure curve, Figure $7 \mathrm{c}$, shows that the simulation method is able to handle short duration dynamics in the fluid system, causing pressure peaks of high amplitude and a duration of approximately $1.5 \mathrm{~ms}$. The results also show that the piston reaches a steady state behaviour after two working cycles, which implies that this method is stable for this simulation model. The FE-results in this case consist of the piston movement and the forces that are acting on the piston.

\subsection{Case 2}

In the second simulation case elastic material properties were used to evaluate deformations, stresses and strains in the structural parts. A tool was also added to simulate the impact from the main piston on the tool and to analyse the responses

Table 2: Estimation of the parasitic inductances for this case

\begin{tabular}{lccccc}
\hline & \multicolumn{5}{c}{ Cavity } \\
Parameter & $\mathrm{A}$ & $\mathrm{B}$ & $\mathrm{C}$ & $\mathrm{D}$ & \\
\hline Volume & $151.1 \cdot 10^{-6}$ & $21.3 \cdot 10^{-6}$ & $7.50 \cdot 10^{-6}$ & $2.61 \cdot 10^{-6}$ & $\mathrm{~m}^{3}$ \\
$Z_{c, f l u i d}$ & $1.76 \cdot 10^{7}$ & $8.35 \cdot 10^{7}$ & $35.6 \cdot 10^{7}$ & $68.2 \cdot 10^{7}$ & $\mathrm{~Pa} \cdot \mathrm{s}^{3}$ \\
Parasitic mass & $7.9 \cdot 10^{-6}$ & $3.2 \cdot 10^{-6}$ & $14 \cdot 10^{-6}$ & $39 \cdot 10^{-6}$ & $\mathrm{~kg}$ \\
Component mass & 11.2 & 11.2 & 0.3 & 0.3 & $\mathrm{~kg}$ \\
\hline
\end{tabular}

in the structure and in the fluid. The system simulation model used is found in Figure 5b. A straight cylindrical part that represents a typical tool for a hydraulic hammer was added to the previous FE-model, see Figure 9. This set-up is used to give a more realistic picture of the structural responses that occurs in the hydraulic percussion unit when the fluid-, and structuralcouplings are accounted for. The far end from the impact surface of the tool was rigidly fixed and the other surfaces are constrained due to symmetry conditions. The elastic material properties stated in Table 1 were used in the FE-model to simulate the elastic behaviour. The mesh for the tool consists of 1040 fully integrated 8-node hexahedral solid elements that gives a total number of elements of 18106 . In order to reduce the noise in the stress results an appropriate amount of structural damping that correspond to a loss factor of $\sim 1.5 \%$, was applied in the FE-model. The time step used here was $1.76 \cdot 10^{-7}$ s to fulfil the Courant-Friedrichs-Lewy condition, i.e. the smallest element size in the FE-model determines the maximum time step [1], resulting in a computational time of 1 hour and 50 minutes. The other parameters in the Hopsan model were the same as in Case 1.

The results, shown in Figure 10, indicate that the percussion unit reaches a steady state condition after only a few working strokes. The values for the piston movement are calculated by averaging over the nodes on the piston impact surface. The piston displacement curve, Figure 10a, shows that the piston follows the same sequence as described in Case 1. The difference is that an elastic impact is simulated in this case, instead of driving the piston against the hydraulic damping mechanism. The impact on the tool occurs at a position of $0.085 \mathrm{~m}$. A stress wave is generated at the piston impact surface at the time of impact, and the noise in the pressure curve, see Figure 10c, after the first impact, represents the stress wave travelling back and forth in the piston. The pressure curve, Figure 10c, is very similar to the curve in Case 1 and it follows the same pattern. The hydraulic pressure in Cavity A shows a rapid variation at the time of impact, see region $\mathrm{N}$ in Figure 10c. At this time the piston 'bounces' on the tool and the piston moves away from the tool. This movement will cause 


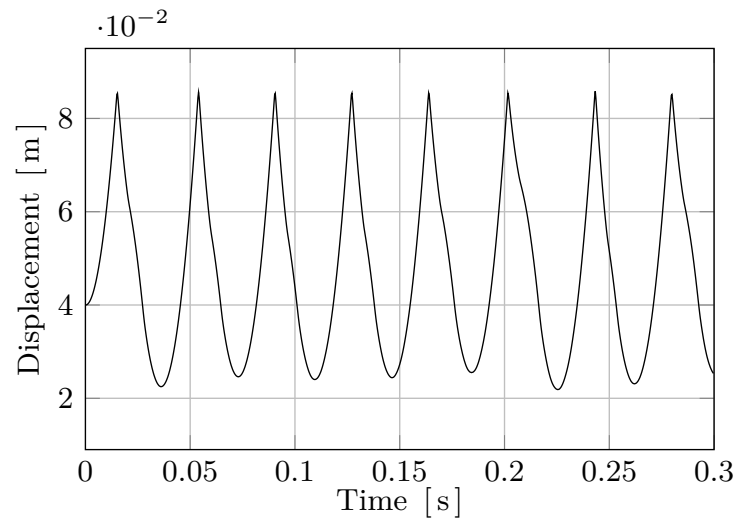

(a) Main piston displacement

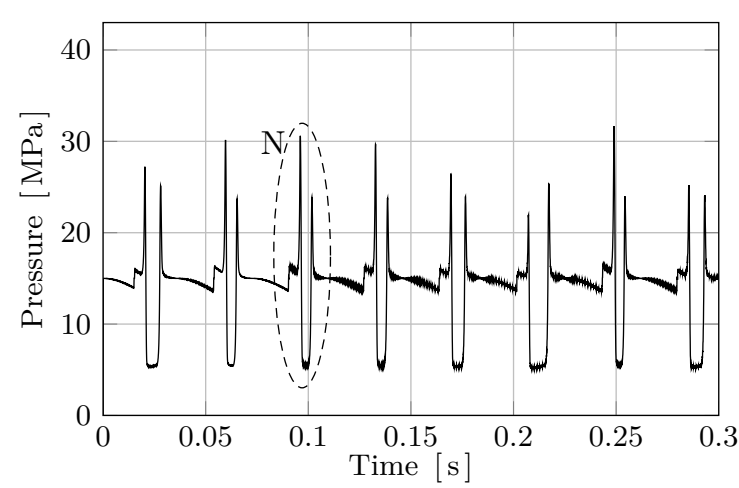

(c) Pressure in piston Cavity A

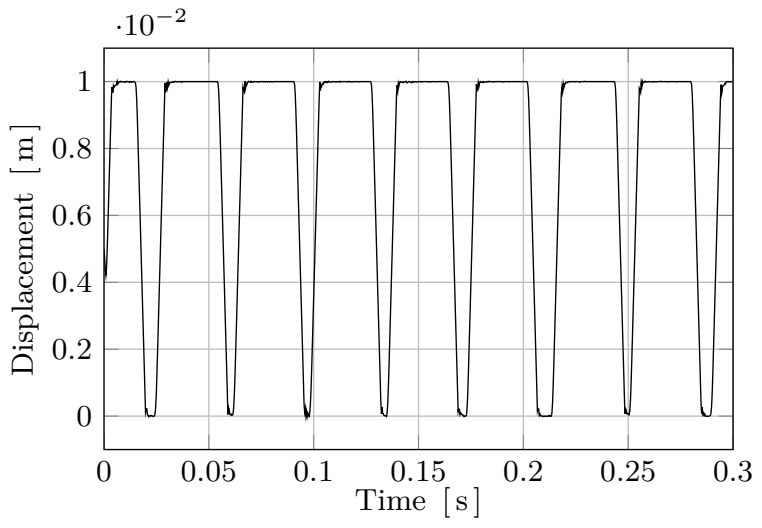

(b) Control valve displacement

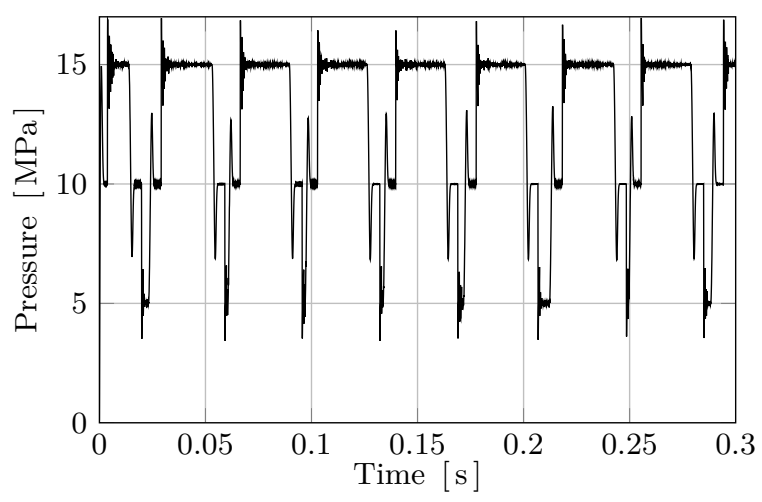

(d) Pressure in valve Cavity $\mathrm{C}$

Figure 10: Simulation results from Case 2. Region $N$ indicates the pressure behaviour at the time of impact

an increased pressure in the fluid contained in Cavity A, since the piston is moving back into the cavity. As the pressure increases in the cavity the fluid force on the piston will also increase, and this will affect the piston movement.

Figure 11 and 12 show some examples of FE-results that are available from the simulation. In this case the equivalent von Mises stress is shown, but also displacements are available. The transient stress curve in Figure 12 exhibits the same behaviour as the pressure curve from Cavity A, cf. Figure 10c.

\section{Discussion}

In this work a previously presented co-simulation method [6] has been extended to include an increased number of submodels for co-simulation, which has been used to increase the number of hydraulic cylinders in the simulation model (FE-model). This has been analysed through two different simulation cases and deviations from a reference model are in this case negligible. No similar work within this area have been found in the literature.

The FMU was configured for two hydraulic cylinders using the co-simulation interface configuration software. This resulted in twice as many ports on the FMU as before and twice as much data were also needed to be communicated over the interface, which will experience twice as high load as evaluated in the previous work. Note that the limiting number of ports was not analysed in this paper. This is of course an important aspect that needs further evaluation.

In Case 1 a co-simulation of a 1D hydraulic system model and a 3D structural FE-model was studied. Here two hydraulic cylinder components were co-simulated, representing the main piston and the control valve of a hydraulic percussion unit, respectively. The comparison between the results from the reference and the co-simulation model shows an identical behaviour, except for a small time shift. The time shift is probably caused by:

- The implementation approximation according to Eq. 4

- Since different solving techniques are used by Hopsan and LS-DYNA, numeric differences are unavoidable, which also will be accumulated throughout the simulation and result in an increasing absolute error

Here a simulation time period of $0.3 \mathrm{~ms}$ was used and during this period 7-8 working cycles were completed, and a time shift error of $\sim 57 \mu$ s for the last working cycle was noticed. From an industrial point of view and for this application, this error can be considered negligible.

Another difference between the models is the modelling of the contact mechanics for the control valve at the mechanical end points. In Hopsan these contacts are perfectly inelastic, which 


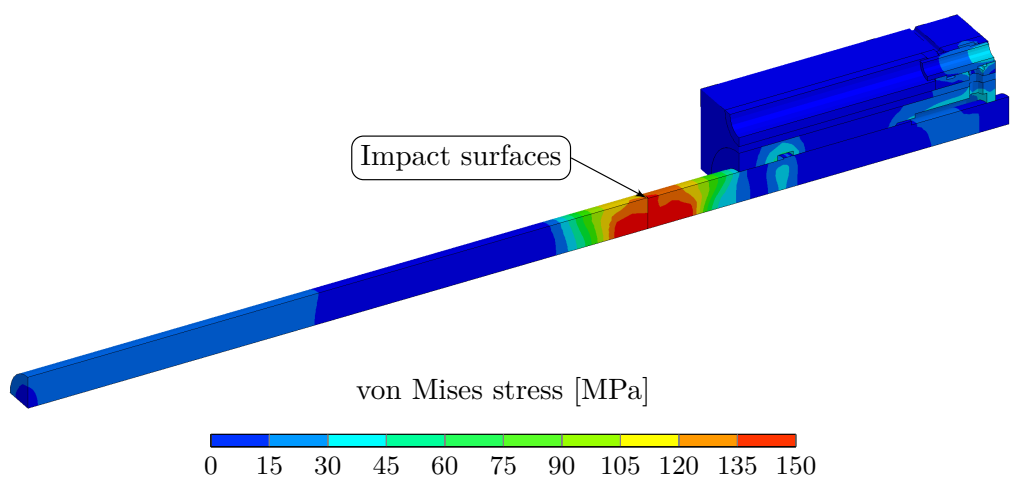

(a) Overall stress at impact

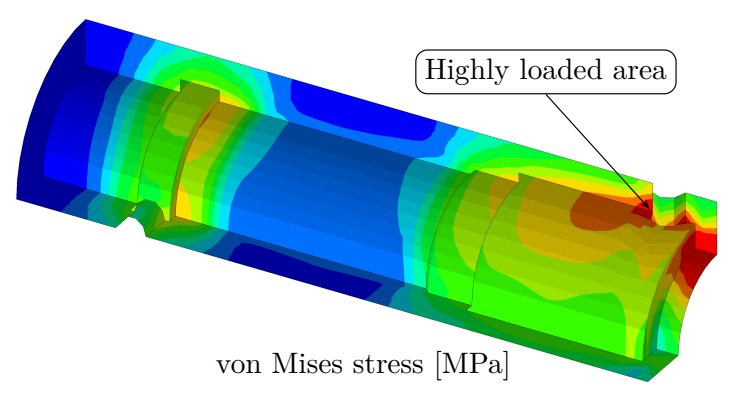

$\begin{array}{lllllllllll}0 & 9 & 18 & 27 & 36 & 45 & 54 & 63 & 72 & 81 & 90\end{array}$

(b) Main piston liner maximum stress

Figure 11: FE results from Case 2, showing a) the von Mises stress in the piston and tool at the time of impact, b) the maximum von Mises stress in the Main piston liner during the analysis, $t=286.3 \mathrm{~ms}$. Notice that different fringe-levels are used

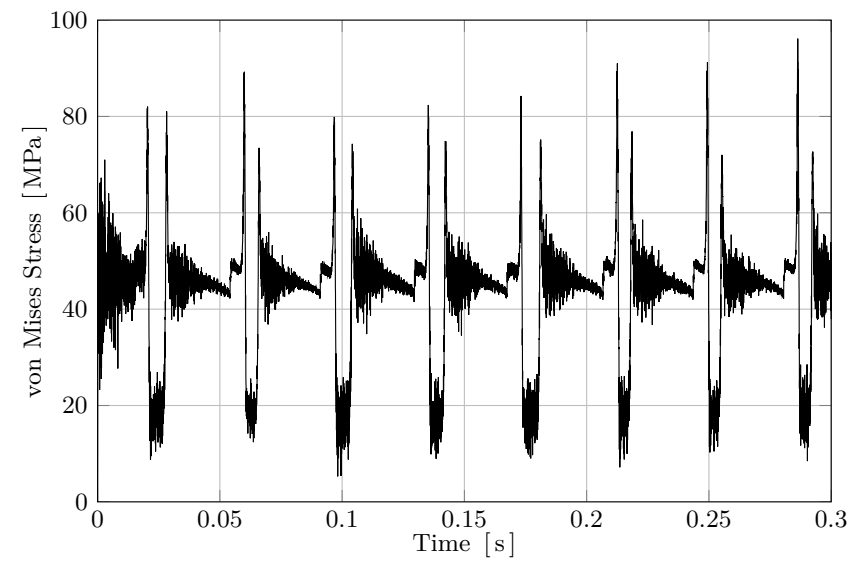

Figure 12: Time history FE-results from Case 2. Stress results from an element at the highly loaded area, shown in Figure 11. The load cycle in the material becomes very clear

means that the control valve will attach to the end point once it is reached. In the co-simulation model the contact mechanics are defined in the LS-DYNA model, and here is the contact of an elastic nature. This means that the momentum of the control valve during the collision will be preserved, and this will cause the control valve to bounce when it hits the end point. In order to reduce the bounces a reasonable amount of contact damping was applied. However, this is not expected to be too unphysical since the collision will occur when oil is present at the contact surfaces, which will reduce the contact forces.

In Case 1 a short duration/high amplitude pressure peak arises in Cavity A, see $t \approx 0.28 \mathrm{~s}$ in Figure $7 \mathrm{c}$. This is an effect of an important mechanism for a hydraulic percussion unit that is captured by the used simulation technique. The identified behaviour indicates that correct and stable results are obtained, and that short duration dynamics are resolved.

Case 2 is meant as a demonstration of the proposed method for an industrial application, closer to reality. No reference model was used for comparison here because no deformable bodies are implemented in Hopsan. The fluid system is the same as before, but the FE-simulation is somewhat more complex due to the deformable bodies and the contact calculations for the impact. The co-simulation coupling method will also be more stressed than in the first case, since the effects of stress waves must be handled. The results from the fluid simulation is similar to the first case. The noise in the pressure curves of Cavity A and C, Figure 10c and 10d, is caused by the stress waves that are travelling back and forth in the components.

The FE-results here chosen consist of deformation and stresses for the whole 3D-geometry and for the simulated time period. The second case shows that the proposed method is capable of simulating the coupled fluid-structure problem where complex dynamic properties, such as mechanical contacts and changing direction of flow, are present. The coupling between the structural- and fluid dynamics that is captured in the proposed co-simulation method is difficult, or impossible, to achieve with decoupled simulation models.

\section{Acknowledgements}

The authors would like to thank the project initiator Erik Sigfridsson and Atlas Copco Construction Tools for funding the project. Furthermore, Mr Peter Nordin and Dr Thomas Borrvall are greatly acknowledged for all their help with developing the co-simulation interface.

\section{Concluding Remarks}

This paper presents a method to co-simulate multiple components of $1 \mathrm{D}$-fluid and 3D-structural models. In order to represent the real mechanisms in a hydraulic percussion unit to a larger extent, this was considered to be a fundamental functionality for a new simulation method. As presented in the previous work [6], the fluid system is modelled in a 1D-system simulation tool and a conventional FE-system is used for the 3D structural simulation. Full 3D results will be available to be used in stress analysis, fatigue assessments or acoustical radiation analysis. The method for simulating the fluid system will capture important system dynamic behaviour without the need for 3D CFD methods, which will give 
a computationally very efficient fluid simulation.

The most important contributions from this work are:

- A co-simulation method for multiple fluid-structure couplings has been developed

- The implemented co-simulation interface, which is based on the FMI-standard and TLM, is shown to also support multiple sub-models for co-simulation

- Full 3D results and time history data from the structural FE-simulation are available for a more complete model of a hydraulic percussion unit

\section{References}

[1] LSTC. LS-DYNA Theory Manual. Livermore Software Technology Corporation, Livermore, USA, 2015.

[2] ANSYS. Inc. ANSYS Multiphysics User's Guide. Canonsburg, Pennsylvania, USA, 2015.

[3] Y Wang, J Feng, B Zhang, and X Peng. Modeling the valve dynamics in a reciprocating compressor based on two-dimensional computational fluid dynamic numerical simulation. Proceedings of the Institution of Mechanical Engineers, Part E: Journal of Process Mechanical Engineering, 227(4):295-308, 2013.

[4] R Campbell and E Paterson. Fluid-structure interaction analysis of flexible turbomachinery. Journal of Fluids and Structures, 27(8):1376-1391, 2011.

[5] R Sinha, C. J. J Paredis, V.-C Liang, and P. K Khosla. Modeling and simulation methods for design of engineering systems. Journal of Computing and Information Science in Engineering, 1(1):84, 2001.

[6] H Andersson, P Nordin, T Borrvall, K Simonsson, D Hilding, M Schill, P Krus, and D Leidermark. A cosimulation method for system-level simulation of fluidstructure couplings in hydraulic percussion units. Engineering with Computers, pages 1-17, 2016.

[7] M Axin, R Braun, A Dell'Amico, B Eriksson, P Nordin, K Pettersson, I Staack, and P Krus. Next generation simulation software using transmission line elements. In Fluid Power and Motion Control, Bath, England, October 2010 .

[8] T Blochwitz, M Otter, J Åkesson, M Arnold, C Clauss, H Elmqvist, M Friedrich, A Junghanns, J Mauss, D Neumerkel, H Olsson, and A Viel. Functional mockup interface 2.0: The standard for tool independent exchange of simulation models. In Proceedings of the 9th International Modelica Conference, pages 173184, Munich, Germany, September 2012.

[9] G Rauch, J Lutz, M Werner, S Gurwara, and P Steinberg. Synergetic 1D-3D-coupling in engine development part i: Verification of concept. Technical report, SAE Technical Paper, 2015.
[10] P Bayrasy, M Burger, C Dehning, I Kalmykov, and M Speckert. Applications for MBS-FEM-coupling with MpCCI using automotive simulation as example. In Proceedings of the 2nd Commercial Vehicle Technology Symposium (CVT 2012), pages 385-394, Kaiserslautern, Germany, March 2012.

[11] R Braun, L Ericsson, and P Krus. Full vehicle simulation of forwarder with semi active suspension using co-simulation. In ASME/BATH 2015 Symposium on Fluid Power and Motion Control, Chicago, USA, October 2015.

[12] A Siemers, D Fritzson, and I Nakhimovski. General meta-model based co-simulations applied to mechanical systems. Simulation Modelling Practice and Theory, 17(4):612-624, 2009.

[13] B Eriksson, P Nordin, and P Krus. Hopsan NG, a C++ implementation using the TLM simulation technique. In The 51st Conference On Simulation And Modelling, Oulu, Finland, 2010.

[14] P Krus, A Jansson, J.-O Palmberg, and K Weddfelt. Distributed simulation of hydromechanical systems. In The Third Bath International Fluid Power Workshop, Bath, England, 1990.

[15] J Larsson and P Krus. Stability analysis of coupled simulation. In ASME 2003 International Mechanical Engineering Congress and Exposition, volume 1, pages 861868, 2003.

[16] D. M Auslander. Distributed system simulation with bilateral delay-line models. Journal of Basic Engineering, 90(2):195-200, 1968.

[17] T. J Viersma. Analysis, Synthesis and Design of Hydraulic Servosystems and Pipelines. Elsevier Scientific Publishing Company, Amsterdam, The Netherlands, 1980.

[18] T Belytschko, W. K Liu, B Moran, and K Elkhodary. Nonlinear finite elements for continua and structures. John Wiley \& Sons, 2013. 